\title{
Registration of 3D Intraoperative MR Images of the Brain Using a Finite Element Biomechanical Model
}

\author{
Matthieu Ferrant ${ }^{1}$, Simon K. Warfield ${ }^{2}$, Arya Nabavi ${ }^{2}$, Ferenc A. Jolesz ${ }^{2}$, and \\ Ron Kikinis $^{2}$ \\ 1 Telecommunications Laboratory, Université catholique de Louvain, Belgium. \\ ${ }^{2}$ Surgical Planning Laboratory, Brigham and Women's Hospital and Harvard Medical School, \\ Boston, USA. \\ \{ferrant, warfield, arya, jolesz,kikinis\}@bwh.harvard.edu
}

\begin{abstract}
We present a new algorithm for the non-rigid registration of 3D Magnetic Resonance (MR) intraoperative image sequences showing brain shift. The algorithm tracks key surfaces (cortical surface and the lateral ventricles) in the image sequence using an active surface algorithm. The volumetric deformation field of the objects the surfaces are embedded in is then inferred from the displacements at the boundary surfaces using a biomechanical finite element model of these objects. The biomechanical model allows us to analyse characteristics of the deformed tissues, such as stress measures. Initial experiments on an intraoperative sequence of brain shift show a good correlation of the internal brain structures after deformation using our algorithm, and a good capability of measuring surface as well as subsurface shift. We measured distances between landmarks in the deformed initial image and the corresponding landmarks in the target scan. The surface shift was recovered from up to $1 \mathrm{~cm}$ down to less than $1 \mathrm{~mm}$, and subsurface shift from up to $6 \mathrm{~mm}$ down to $3 \mathrm{~mm}$ or less.
\end{abstract}

\section{Introduction}

The increased use of image guided surgery systems for neurosurgery has brought to prominence the problem of brain shift, the deformation the brain undergoes after craniotomy, as well as deformations due to tumor resection. These deformations can significantly diminish the accuracy of neuronavigation systems, and it is therefore of great importance to be able to quantify and analyse these phenomena. The subject has recently lead to considerable interest in the medical image analysis community $[1,2,3$, $4,5,6,7,8,9]$.

Most of the work that has been done in the field of intraoperative volumetric image alignment is mainly based on image related criteria $[10,8,4]$. Physical deformation models have also been proposed to constrain a deformation field computed from image data using elastic [11, 12] or even viscous fluid deformation models [13, 14]. However, these models do not account for the actual material characteristics of the brain, because the matching is done minimizing an energy measure that consists of a weighted sum of an image similarity term and a relaxation term representing the potential energy of a physical body (e.g. elastic). Therefore, the actual physics of the phenomenon cannot not be properly captured by these models.

There has also been a significant amount of work directed towards simulation using models driven by physics-based forces such as gravity. Skrinjar et al. [15, 7] have 
proposed a model consisting of mass nodes interconnected by Kelvin models to simulate the behavior of brain tissue under gravity, with boundary conditions to model the interaction of the brain with the skull. Miga et al. [3, 5, 6] proposed a Finite Element (FE) model based on consolidation theory where the brain is modeled as an elastic body with an intersticial fluid. They also use gravity induced forces, as well as experimentally determined boundary conditions.

Even though these models are very promising, it remains difficult to accurately estimate all the forces and boundary conditions that interact with the model.

It is only recently that biomechanical models have been explicitly proposed to constrain the deformation of images $[16,9]$. Currently, the drawback of such methods is that they either require user intervention, or another means to compute the forces (or correspondances) applied to the model. Another drawback is that these later methods have only been applied to 2D images thereby limiting the clinical utility and the possibility to efficiently assess the accuracy of the method.

Our ultimate goal is to be able to do prediction of deformation during surgery with the goal of improving intraoperative navigation and tumor resection, and of reducing the amount of intraoperative imaging that is necessary. To be able to do this, one first needs to validate the non-rigid deformation model. Intraoperative MRI provides excellent contrast and spatial resolution, which makes it an ideal testbed for developing and validating nonrigid deformation methods.

We propose a new integrated approach that uses surface-based correspondences to drive a biomechanical model of the brain instead of using estimates of forces that are often difficult to accurately determine. The correspondances are computed using one or multiple active surfaces that are deformed onto the target image. The correspondances between landmark surfaces in the preoperative and intraoperative scans provide an implicit way to compute the forces the model has undergone.

\section{Theory}

So far, we have limited our model to linear elasticity, as it has been shown that soft tissue deformation can be modeled quite accurately using linear elasticity in the case of small strains $[17,18]$. However, our algorithm can easily be used with other constitutive materials such as viscous fluids, etc. The following sections will successively review the theory of finite element modeling and address the FE meshing issue, explain how we have used these principles to solve active surface problems, as well as the way we use it for computing a biomechanical volumetric deformation field.

\subsection{Finite Element Model}

Assuming a linear elastic continuum with no initial stresses or strains, the potential energy of an elastic body submitted to externally applied forces can be expressed as [19] ${ }^{1}$ :

$$
E=\frac{1}{2} \int_{\Omega} \boldsymbol{\sigma}^{T} \boldsymbol{\epsilon} d \Omega+\int_{\Omega} \mathbf{F u} d \Omega
$$

\footnotetext{
${ }^{1}$ Superscript $\mathrm{T}$ designs the transpose of a vector or a matrix
} 
where $\mathbf{u}=\mathbf{u}(x, y, z)$ is the displacement vector, $\mathbf{F}=\mathbf{F}(x, y, z)$ the vector representing the forces applied to the elastic body (forces per unit volume, surface forces, or forces concentrated at the nodes), and $\Omega$ the body on which one is working. $\epsilon$ is the strain vector, defined as

$$
\boldsymbol{\epsilon}=\left(\frac{\partial \mathbf{u}}{\partial x}, \frac{\partial \mathbf{u}}{\partial y}, \frac{\partial \mathbf{u}}{\partial z}, \frac{\partial \mathbf{u}}{\partial x}+\frac{\partial \mathbf{u}}{\partial y}, \frac{\partial \mathbf{u}}{\partial y}+\frac{\partial \mathbf{u}}{\partial z}, \frac{\partial \mathbf{u}}{\partial x}+\frac{\partial \mathbf{u}}{\partial z}\right)^{T}=\mathbf{L u}
$$

and $\sigma$ the stress vector, linked to the strain vector by the constitutive equations of the material. In the case of linear elasticity, with no initial stresses or strains, this relation is described as

$$
\boldsymbol{\sigma}=\left(\sigma_{x}, \sigma_{y}, \sigma_{z}, \tau_{x y}, \tau_{y z}, \tau_{x z}\right)^{T}=\mathbf{D} \boldsymbol{\epsilon}
$$

where $\mathbf{D}$ is the elasticity matrix characterizing the properties of the material [19].

This equation is valid whether one is working with a surface or a volume. We model our active surfaces, which represent the boundaries of the objects in the image, as elastic membranes, and the surrounding and inner volumes as 3D volumetric elastic bodies.

Within a finite element discretization framework, an elastic body is approximated as an assemblage of discrete finite elements interconnected at nodal points on the element boundaries. This means that the volumes to be modeled need to be meshed, i.e., divided into elements. In [9], Hagemann et al. propose to use the pixels of the image as basic elements of his FE mesh. This approach does not take advantage of the intrinsic formulation of FE modeling, which assumes that the mechanical properties are constant over the element, suggesting that one can use elements covering several image pixels. Also, when performing computations in $3 \mathrm{D}$, which is eventually what is needed for medical applications, the amount of degrees of freedom will be far too large (for a typical 256x256x60 intraoperative MRI, this means about 12 million degrees of freedom !) to perform efficient computations in a reasonable time, even on high performance computing equipment.

Most available meshing software packages do not allow meshing of multiple objects [20,21], and are usually designed for regular and convex objects, which is often not the case for anatomical structures. Therefore, we have implemented a tetrahedral mesh generator specifically suited for labeled 3D medical images. The mesher can be seen as the volumetric counterpart of a marching tetrahedra surface generation algorithm. A detailed description of the algorithm can be found in [22]. The resulting mesh structure is built such that for images containing multiple objects, a fully connected and consistent tetrahedral mesh is obtained with for every cell, a given label corresponding to the object the cell belongs to. Therefore, different biomechanical properties and parameters can easily be assigned to the different cells or objects composing the mesh. Boundary surfaces of objects represented in the mesh can be extracted from the mesh as triangulated surfaces, which is very convenient for running an active surface algorithm.

The continuous displacement field $\mathbf{u}$ within each element is a function of the displacement at the nodal points of the element $\mathbf{u}_{i}^{e l}$ weighted by its shape functions $N_{i}^{e l}=$ $N_{i}^{e l}(x, y, z)$.

$$
\mathbf{u}=\sum_{i=1}^{N_{\text {nodes }}} N_{i}^{e l} \mathbf{u}_{i}^{e l}
$$


The elements we use are tetrahedra $\left(N_{\text {nodes }}=4\right)$ for the volumes and triangles for the membranes $\left(N_{\text {nodes }}=3\right)$, with linear interpolation of the displacement field. Hence, the shape function of node $i$ of element $e l$ is defined as: $N_{i}^{e l}=K\left(a_{i}^{e l}+b_{i}^{e l} x+\right.$ $\left.c_{i}^{e l} y+d_{i}^{e l} z\right)$, where $K=\frac{1}{6 V^{e l}}$ for a tetrahedron, and $K=\frac{1}{2 S^{e l}}$ for a triangle. The computation of $V^{e l}, S^{e l}$ (volume, surface of $e l$ ) and other constants is detailed in [19].

For every node $i$ of each element $e l$, we define the matrix $\mathbf{B}_{i}^{e l}=\mathbf{L}_{i} N_{i}^{e l}$. The function to be minimized at every node $i$ of each element $e l$ can thus be expressed as :

$$
E\left(\mathbf{u}_{i}^{e l}\right)=\int_{\Omega} \sum_{j=1}^{N_{\text {nodes }}} \mathbf{u}_{i}^{e l^{T}} \mathbf{B}_{i}^{e l^{T}} \mathbf{D B}_{j}^{e l} \mathbf{u}_{j}^{e l}+\mathbf{F} N_{i}^{e l} \mathbf{u}_{i}^{e l} d \Omega
$$

We seek the minimum of this function by solving for $\frac{d E\left(u_{i}^{e l}\right)}{d u_{i}^{e l}}=0$. Equation (5) then becomes :

$$
\int_{\Omega} \sum_{j=1}^{N_{\text {nodes }}} \mathbf{B}_{i}^{e l}{ }^{T} \mathbf{D B}_{j}^{e l} \mathbf{u}_{j}^{e l} d \Omega=-\int_{\Omega} \sum_{j=1}^{N_{\text {nodes }}} \mathbf{F} N_{i}^{e l} d \Omega
$$

This last expression can be written as a matrix system for each finite element:

$$
\mathbf{K}^{e l} \mathbf{u}^{e l}=-\mathbf{F}^{e l}
$$

Matrices $\mathbf{K}^{e l}$ and vector $\mathbf{F}^{e l}$ are defined as follows: $\mathbf{K}_{i, j}^{e l}=\int_{\Omega} \mathbf{B}_{i}^{e^{T}} \mathbf{D B}_{j}^{e l} d \Omega, \mathbf{F}_{j}^{e l}=$ $\int_{\Omega} \mathbf{F} N_{i}^{e l} d \Omega$; where every element $i, j$ refers to pairs of nodes of the element $e l$ ( $i$ and $j$ range from 1 to 4 for a tetrahedron -1 to 3 for a triangle). $\mathbf{K}_{i, j}^{e l}$ and are 3 by 3 matrices, $\mathbf{F}_{j}^{e l}$ is a 3 by 1 vector. The 12 by 12 ( 9 by 9 for a triangle) matrix $\mathbf{K}^{e l}$, and the vector $\mathbf{F}^{e l}$ are computed for each element and are then assembled in a global system $\mathbf{K u}=-\mathbf{F}$, the solution of which will provide us with the deformation field corresponding to the global minimum of the total energy.

We now have constitutive equations that model surfaces as elastic membranes and volumes as elastic bodies.

\subsection{Active Surface Algorithm}

The active surface algorithm deforms the boundary surface of an object in one image of the sequence towards the boundary of the same object in the next image of the sequence. The surface is modeled as an elastic membrane, which we deform iteratively onto the target scan by applying image-derived forces. As proposed in [23], the temporal variation of the surface can be discretized using finite differences, provided the time step $\tau$ is small enough. Using the previously obtained relation for elastic bodies (see eq. 7 ), this yields the following semi-implicit iterative equation ${ }^{2}$ :

$$
\frac{\mathbf{u}^{t}-\mathbf{u}^{t-1}}{\tau}+\mathbf{K} \mathbf{u}^{t}=-\mathbf{F}^{\mathbf{u}^{t-1}}
$$

Classically, the image force $\mathbf{F}$ is computed as a decreasing function of the gradient so as to be minimized at the edges of the image. To increase the robustness and the convergence rate of the process, we have computed the forces as a steepest gradient descent on a euclidean distance map we efficiently compute from the target object [24]. More details about our active surface algorithm can be found in [25].

\footnotetext{
${ }^{2}$ Superscript $t$ refers to the current temporal iteration.
} 


\subsection{Inferring Volumetric Deformations from Surface Deformations}

The deformation field obtained for the boundary surfaces is then used in conjunction with the volumetric model to infer the deformation field inside and outside the boundary surfaces. The idea is to apply forces to the boundary surfaces that will produce the same displacement field at the boundary surfaces that was obtained with the active surface algorithm. The volumetric biomechanical model will then compute the deformation of the surrounding nodes in the mesh.

Let $\widetilde{\mathbf{u}}$ be the vector representing the displacement to be imposed at the boundary nodes. Hence, the forces (see eq. 7) needed to impose these displacements to the volume can be expressed as :

$$
\mathbf{F}=\mathbf{K} \widetilde{\mathbf{u}}
$$

The solution of the equilibrium equations with these forces will provide us with the displacement at all the nodes in the volumetric mesh with the imposed displacements at the nodes of the boundary surfaces delimiting the objects represented in the mesh. Biomechanical parameters such as the stress tensors can then be derived from the displacements at the nodes using the stress-strain relationship (eq. 3) for every node $i$ :

$$
\sigma_{i}=\sum_{e l \mid i \in e l} \mathbf{D} \epsilon_{i}=\sum_{e l \mid i \in e l} \mathbf{D L}_{i} N_{i}^{e l} \mathbf{u}_{i}
$$

\section{Experiments}

\subsection{FE Model Generation}

To build our brain model, we segmented the brain out of the initial intraoperative MRI using our directional watershed algorithm [26]. The volume was further simplified using mathematical morphology to obtain a smooth surface. Figure 1 shows cuts through a sample tetrahedral mesh of the brain overlayed on the corresponding initial image. Note that the mesh has been adaptively refined in the neighborhood of the lateral ventricles, so as to ensure sufficient resolution of the surfaces for the active surface algorithm.
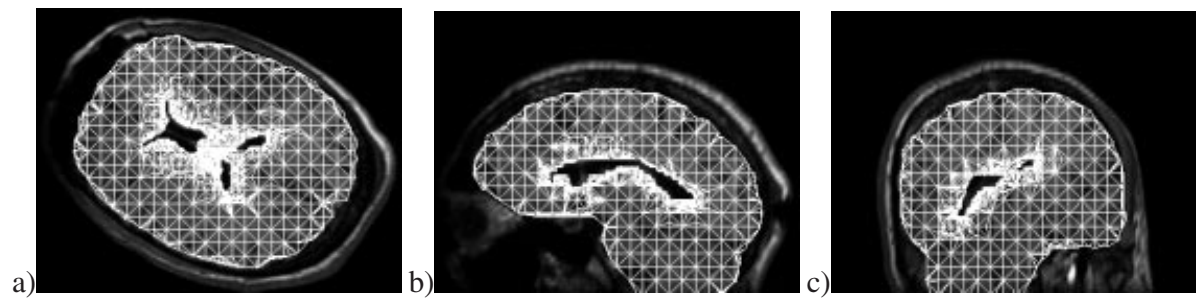

Fig. 1. Axial (a), sagittal (b), and coronal (c) cuts through tetrahedral mesh of the brain overlayed on corresponding cuts through preoperative image.

An isotropic linear elastic material is characterized by two parameters: Young's elasticity modulus $E$ and Poisson's ratio $\nu$ [19]. They determine the elastic behavior of the object. The choice of these values is of course critical to the reliability of a physics 
based deformation model. Their determination has not been addressed very consistently in the literature as the coefficients used often differ significantly from study to study and do not always include the physical units of the values. Recently, Hagemann et. al.[9] published a comparative study of brain elasticity coefficients proposed by different authors, and came to the conclusion that for their application, the only comparable and meaningful values presented by other authors are the ratios of the coefficients for brain and skull. Since we are only interested in modeling the brain, and not the skull, we have chosen to use the parameters Miga et al. [6] obtained with in-vivo experiments instead $(E=3 k P a, \nu=0.4)$.

We have implemented our own FE algorithm, both for the active surface matching and for the volumetric biomechanical deformation. The assembly and solving of the linear matrix systems have been parallelized using the PETSc library [27]. The entire deformation algorithm, using a mesh with approximately one hundred thousand tetrahedra, only takes about 30 minutes on a Sun Ultra $10440 \mathrm{MHz}$ workstation. Using 4 CPUs, the computation time can be reduced to 10 minutes. The average size of the edges of the larger tetrahedra was approximately $15 \mathrm{~mm}$, while the smallest tetrahedra (in the neighborhood of the ventricles) had edges of $1.5 \mathrm{~mm}$. However, it must be noted that the meshing algorithm yields even smaller tetrahedra in the neighborhood of boundary edges. Before we applied our algorithm, the images have been aligned using our rigid registration algorithm based upon maximization of mutual information [28] so as to account for patient movement within the magnet during the operation.

\subsection{Active Surface Matching}

The active surfaces are extracted from the intraoperative scan at the start of surgery, before opening the dura mater (see Figure 2a), and deformed towards the brain in a later intraoperative image (see Figure $2 b$ ). One can very clearly observe that the deformation of the cortical surface is happening in the direction of gravity and is mainly located where the dura was removed. Also, one can observe a shift, as well as a contraction of the lateral ventricles. Figure 4 shows the 3D surface deformation field the brain and the ventricles have undergone. One can very well observe that the shift is mainly affecting the left part of the ventricles, while the displacement of the lower parts is mostly due to volume loss.
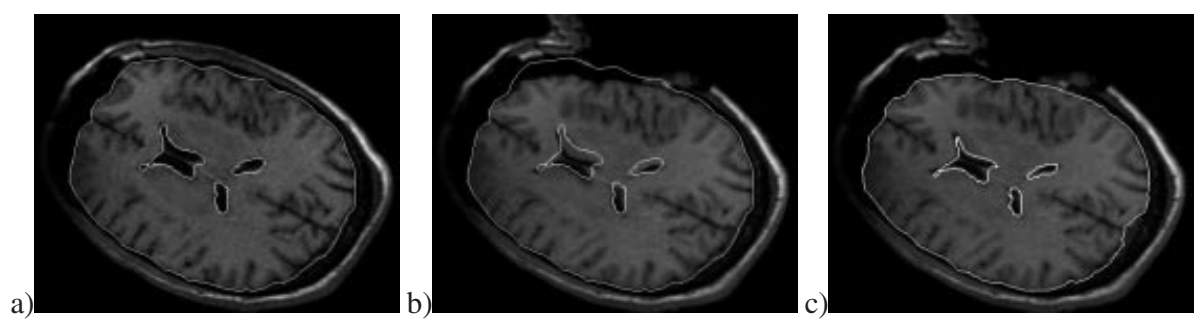

Fig. 2. Axial cut through initial (a and b) and deformed (c) active surfaces overlayed on corresponding slice of (a)initial; b,c)target) intraoperative MR image 


\subsection{Volumetric FE Deformation}

The deformation field obtained with the active surface algorithm is then used as input for our biomechanical FE model. The algorithm yields a deformation vector for every node of the mesh. These displacements can then be interpolated back onto the image grid using the shape functions within every element of the FE mesh (see eq. 4). Figure 3 shows a slice of the deformed image as well as the image of the difference with the target. One can observe that the algorithm captured the surface shift and the ventricular thinning very accurately. The gray-level mean square difference between the target scan and the deformed original scan on the image regions covered by the mesh went down from 15 to 3 . However, one can also notice that the left ventricle (lower one on the Figure) was not able to fully capture the thinning. This is due to the approximate model of the lateral ventricles we used in this experiment.
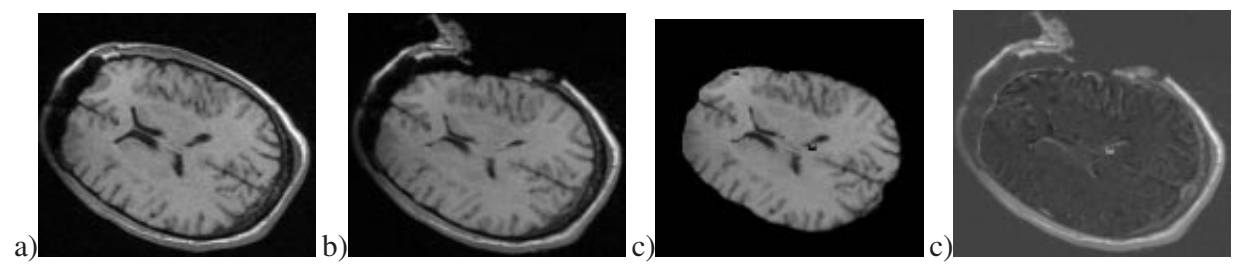

Fig. 3. Slice 29 of a) initial scan b) target scan c)initial scan deformed using our algorithm d)difference between target scan and deformed initial scan.

Figure 6 shows orthogonal cuts through the target intraoperative scan with transparently overlayed color-coding of the intensity of the deformation field. The arrows show the actual displacement of the nodes of the mesh. The extremely dense vector field in the neighborhood of the lateral ventricles is due to the adaptive refinement of the mesh at these locations.

Figure 5a shows the obtained deformation field overlayed on a slice of the initial scan, and Figure 5b shows the same slice of the initial scan deformed with the obtained deformation field. Several landmarks have also been placed on the initial scan (green crosses) and deformed onto the target scan (red crosses), and these last landmarks have also been overlayed on the target scan for comparison with the actual deformed anatomy.

Similar landmarks as those shown on Figure 5 have been placed on 4 different slices where the shift was most visible, and the distance between deformed landmarks and target landmarks (not represented here for better visibility) have been measured. The surface based landmarks on the deformed scan were within $1 \mathrm{~mm}$ of the landmarks on the target intraoperative scan. The errors between the landmarks placed in between the midsagittal plane and the cortical surface were within $2-3 \mathrm{~mm}$ from the actual landmarks. The largest errors were observed at the level of the mid-sagittal plane and ventricles, which can be explained by the fact that the surface matching of the ventricles was not perfect. Nevertheless, the algorithm reduced the distance between landmarks in the initial and the target scans from up to almost $1 \mathrm{~cm}$ to less than $1 \mathrm{~mm}$ for the surface-based landmarks, and from up to $6 \mathrm{~mm}$ to $3 \mathrm{~mm}$ or less for the sub-surface landmarks. 


\section{Discussion and Conclusion}

We have presented a biomechanical FE deformable model for the registration of image sequences showing brain shift. The biomechanical model is driven by imposing displacements to key boundary surfaces. The displacements at the boundary surfaces are computed using an active surface algorithm.

The algorithm provides us with a physically realistic deformation field and also allows us to inspect the characteristics of the deformed objects. This can be very useful for the inspection of stresses induced by the deformation of certain objects on their surroundings.

Our algorithm was able to track the surface shift the brain undergoes very accurately and partially correct for the subsurface shift. In the experiment we presented, the brain was considered to be an homogeneous elastic body. Further improvements of the algorithm include the modeling of different intracranial structures, and the assignment of the corresponding material properties. Also, we plan to investigate if changing the elasticity coefficients and introducing anisotropy (by modifying the elasticity matrix $\mathbf{D}$ appropriately) can improve the non-rigid registration.

\section{Acknowledgments}

Matthieu Ferrant is working towards a Ph.D. degree with a grant from the Belgian FRIA. This research was supported (in part) by NIH P41 RR13218-01, NIH P01 CA67165-03, and NIH R01 RR11747-01A. We thank Peter McL. Black for the opportunity and his patience, that enabled us to acquire the data necessary for our study.

\section{References}

[1] R.D. Bucholz, D.D. Yeh, J. Trobaugh, L.L. McDurmont, C.D. Sturm, C. Baumann, J.M. Henderson, A. Levy, and P. Kessman. The correction of stereotactic inaccuracy caused by brain shift using an intraoperative ultrasound device. In J. Troccaz, E. Grimson, and R. Mösges, editors, CVRMed-MRCAS '97, pages 459-466. Springer-Verlag, Berlin, 1997.

[2] C.R. Maurer, D.L.G. Hill, A.J. Martin, H. Liu, M. McCue, D. Rueckert, Lloret D., W.A. Hall, R.E. Maxwell, D.J. Hawkes, and C.L. Truwit. Investigation of intraoperative brain deformation using a 1.5 tesla interventional $\mathrm{mr}$ system: Preliminary results. IEEE Transactions on Medical Imaging, 17:817-825, 1998.

[3] D.D. Paulsen, M.I. Miga, F.E. Kennedy, P.J. Hoopes, A. Hartov, and D.W. Roberts. A Computational Model for Tracking Subsurface Tissue Deformation During Stereotactic Neurosurgery. IEEE Transactions on Biomedical Engineering, 46(2):213-225, February 1999.

[4] D.L.G. Hill, C.R. Maurer, A.J. Martin, S. Sabanathan, W.A. Hall, D.J. Hawkes, D. Rueckert, and C.L. Truwit. Assessment of intraoperative brain deformation using interventional $\mathrm{mr}$ imaging. In Berlin Springer-Verlag, editor, MICCAI '99, pages 910-919, 1999.

[5] M.I. Miga, K.D. Paulsen, J.M. Lemery, S. D. Eisner, A. Hartov, F.E. Kennedy, and D.W. Roberts. Model-updated image guidance: Initial clinical experiences with gravity-induced brain deformation. IEEE Transactions on Medical Imaging, 18(10):866-874, October 1999.

[6] M.I. Miga, K.D. Paulsen, P.J. Hoopes, F.E. Kennedy, A. Hartov, and D.W. Roberts. In vivo quantification of a homogeneous brain deformation model for updating preoperative images during surgery. IEEE Transactions on Medical Imaging, 47(2):266-273, February 2000.

[7] O.M. Skrinjar and J.S. Duncan. Real time 3d brain shift compensation. In IPMI '99, 1999.

[8] N. Hata, A. Nabavi, S. Warfield, W.M. Wells, R. Kikinis, and Jolesz F.A. A volumetric optical flow method for measurement of brain deformation from intraoperative magnetic resonance images. In C.Taylor and A.Colchester, editors, MICCAI '99, Lecture Notes in Computer Science, pages 928-935. Springer-Verlag, 1999. 
[9] A. Hagemann, Rohr K., H.S. Stiel, U. Spetzger, and Gilsbach J.M. Biomechanical Modeling of the Human Head for Physically Based, Non-Rigid Image Registration. IEEE Transactions on Medical Imaging, 18(10):875-884, October 1999.

[10] N. Hata, R. Dohi, S. Warfield, W.M. Wells, R. Kikinis, and Jolesz F.A. Multimodality deformable registration of pre- and intraoperative images for MRI-guided brain surgery. In MICCAI '98, Lecture Notes in Computer Science, pages 1067-1074. Springer-Verlag, 1998.

[11] R. Bajcsy and S. Kovacic. Multi-resolution Elastic Matching. Computer Vision, Graphics, and Image Processing, 46:1-21, 1989.

[12] C. Davatzikos. Spatial Transformation and Registration of Brain Images Using Elastically Deformable Models. Computer Vision and Image Understanding, 66(2):207-222, May 1997.

[13] G.E. Christensen, S.C. Joshi, and M.I. Miller. Volumetric Transformation of Brain Anatomy. IEEE Transactions on Medical Imaging, 16(6):864-877, December 1997.

[14] M. Bro-Nielsen and C. Gramkow. Fast Fluid Registration of Medical Images. In Visualization in Biomedical Computing (VBC'96), pages 267-276, 1996.

[15] O. Skrinjar, D. Spenser, and J. Duncan. Brain Shift Modeling for use in Neurosurgery. In MICCAI'98, pages 641-649. Springer-Verlag, 1998.

[16] S.K. Kyriacou, C. Davatzikos, S.J. Zinreich, and R.N. Bryan. Nonlinear elastic registration of brain images with tumor pathology using a biomechanical model. IEEE Transactions on Medical Imaging, 18(7):580-592, july 1999.

[17] Y.C. Fung. Biomechanics: Mechanical Properties of Living Tissues. Springer-Verlag, Berlin, Germany, 1993.

[18] E.K. Walsh and A. Schettini. Brain tissue elasticity and CSF elastance. Neurolog. Research, 12:123-127, June 1990.

[19] O.C. Zienkewickz and R.L. Taylor. The Finite Element Method. McGraw Hill Book Co., 1987.

[20] Will Schroeder, Ken Martin, and Bill Lorensen. The Visualization Toolkit: An ObjectOriented Approach to 3D Graphics. Prentice Hall PTR, New Jersey, second edition, 1998.

[21] B. Geiger. Three dimensional modeling of human organs and its application to diagnosis and surgical planning. Technical Report 2105, INRIA, 1993.

[22] M. Ferrant, S.K. Warfield, C.R.G. Guttman, F.A. Jolesz, and R. Kikinis. 3D Image Matching Using a Finite Element Based Elastic Deformation Model. In Taylor C. and Colchester A., editors, MICCAI '99, volume 1679, pages 202-209. Springer-Verlag, 1999.

[23] L.D. Cohen and Cohen I. Finite Element Methods for Active Contour Models and Balloons for 2D and 3D Images. IEEE Transactions on Pattern Analysis and Machine Intelligence, 15:1131-1147, 1993.

[24] O. Cuisenaire and B. Macq. Fast Euclidean Distance Transformation by Propagation Using Multiple Neighborhoods. Computer Vision and Image Understanding, 76(2):163-172, November 1999.

[25] M. Ferrant, O. Cuisenaire, and B. Macq. Multi-Object Segmentation of Brain Structures in 3D MRI Using a Computerized Atlas. In SPIE Medical Imaging '99, volume 3661-2, pages 986-995, 1999.

[26] J.P. Thiran, V. Warscotte, and B. Macq. A Queue-based Region Growing Algorithm for Accurate Segmentation of Multi-Dimensional Images. Signal Processing, 60:1-10, 1997.

[27] S. Balay, W.D. Gropp, L. Curfman McInnes, and B.F. Smith. PETSc 2.0 for MPI - Portable, Extensible Toolkit for Scientific Computations. http://www.mcs.anl.gov/petsc, 1998.

[28] P. Viola and W.M. Wells. Alignment by Maximization of Mutual Information. International Journal of Computer Vision, 24(2):137-154, 1997. 
a)
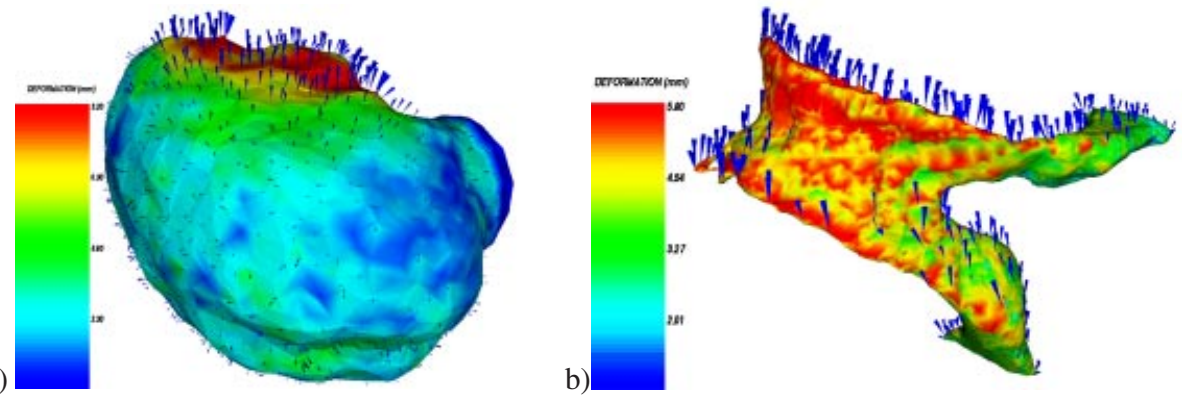

Fig. 4. 3D surface renderings of active surfaces(a) brain surface, b)lateral ventricles) with colorcoded intensity of deformation field
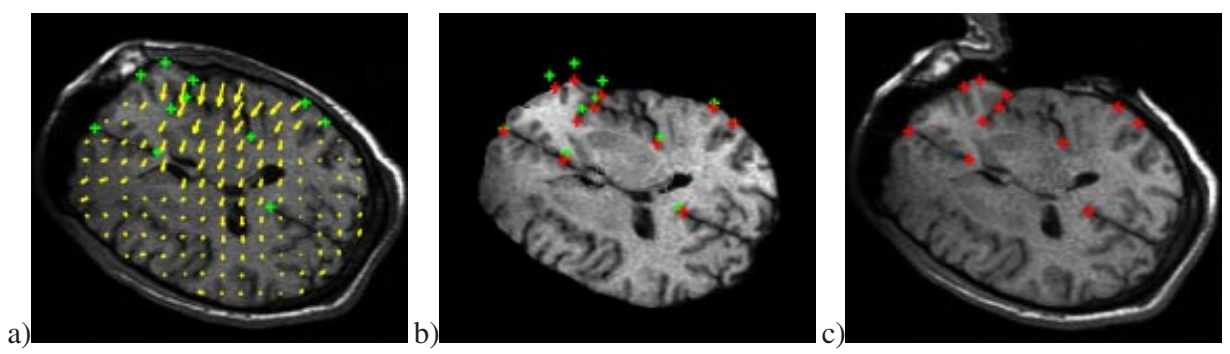

Fig. 5. a)Volumetric deformation field and initial landmarks (green) overlayed on initial intraoperative image slice, b) Same slice of deformed initial image with deformed initial landmarks (red), c) Same slice of target image with deformed landmarks

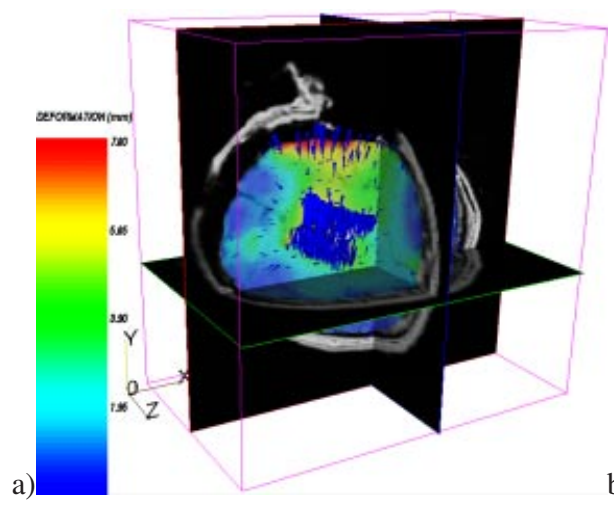

b)

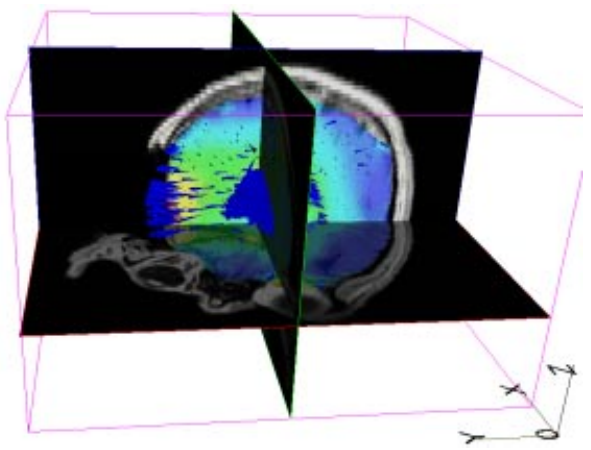

Fig. 6. 3D Volumetric Deformation field (downsampled 12x, scaled 2x) with orthogonal cuts through target intraoperative MR image and transparently overlayed color coded intensity of the deformation field a)Axial view, gravity is downwards. b)Coronal view, gravity goes from left to right 